\title{
EFFECT OF pH ON KRAFT LIGNIN DEPOLYMERISATION IN SUB-CRITICAL
}

\section{WATER}

$\underline{\text { Tallal Belkheiri }}^{a^{*}}$, Cecilia Mattsson $^{b}$, Sven-Ingvar Andersson ${ }^{a}$, Lars Olausson $^{c}$, Lars-Erik Aimand $^{a}$, Hans Theliander ${ }^{b}$ Lennart Vamling ${ }^{a}$

${ }^{a}$ Chalmers University of Technology, Department of Energy and Environment, SE-412 96 Gothenburg, Sweden

${ }^{b}$ Chalmers University of Technology, Department of Chemistry and Chemical Engineering, SE-412 96 Gothenburg, Sweden

${ }^{c}$ Valmet AB, Box 8734, SE-402 75 Gothenburg, Sweden

* Tallal.Belkheiri@,chalmers.se (Corresponding author)

Table S1. Distribution (\% wt.) of compounds identified by GC-MS in the diethyl ether soluble fraction (light oil).

\begin{tabular}{lcccccc}
\hline Compound & \multicolumn{7}{c}{ Experiment } \\
\hline Anisoles & $\mathbf{A}$ & $\mathbf{B}$ & $\mathbf{C}$ & $\mathbf{D}$ & $\mathbf{E}$ & $\mathbf{F}$ \\
Phenol & 18.3 & 17.0 & 18.5 & 16.9 & 19.9 & 20.5 \\
Alkyl phenols & 39.3 & 41.0 & 41.5 & 42.4 & 35.2 & 33.5 \\
Guaiacols & 17.9 & 18.9 & 19.0 & 22.0 & 26.6 & 28.5 \\
Acetyl phenols & 8.4 & 6.2 & 5.9 & 4.7 & 3.6 & 3.2 \\
Catechols & 0.4 & 0.5 & 0.4 & 0.4 & 0.5 & 0.5 \\
Xanthenes & 1.9 & 1.6 & 1.9 & 1.7 & 2.3 & 2.1 \\
Phenolic dimers & 2.3 & 2.3 & 2.0 & 2.0 & 2.3 & 2.3 \\
Aromatics(condensed rings) & 8.7 & 9.3 & 8.7 & 8.1 & 8.3 & 8.3 \\
Retene & 0.8 & 1.3 & 0.8 & 0.7 & 0.5 & 0.4 \\
Dehydroabietic acid & 0.8 & 0.7 & 0.5 & 0.7 & 0.6 & 0.6 \\
& 1.1 & 0.9 & 0.6 & 0.4 & 0.2 & 0.2 \\
\hline
\end{tabular}


Table S2. Mass fraction (\% wt.) of the compounds identified by GCMS in the water phase (WSO).

\begin{tabular}{lcccccc}
\hline Compound & \multicolumn{6}{c}{ Experiment } \\
& A & B & C & D & E & F \\
\hline Anisoles & 0.049 & 0.028 & 0.019 & 0.020 & 0.029 & 0.030 \\
Phenol & 1.651 & 1.646 & 1.460 & 1.325 & 1.543 & 1.490 \\
Alkyl phenols & 0.209 & 0.199 & 0.186 & 0.178 & 0.292 & 0.294 \\
Guaiacols & 0.074 & 0.053 & 0.048 & 0.030 & 0.031 & 0.027 \\
Acetyl phenols & 0.017 & 0.013 & 0.022 & 0.016 & 0.050 & 0.045 \\
Catechols & 0.229 & 0.236 & 0.215 & 0.195 & 0.294 & 0.310 \\
Phenolic dimers & 0.011 & 0.009 & 0.012 & 0.010 & 0.033 & 0.034 \\
Aromatics(condensed rings) & 0.013 & 0.018 & 0.028 & 0.013 & 0.033 & 0.024 \\
\hline Total mass fraction & $\mathbf{2 . 2 5 4}$ & $\mathbf{2 . 2 0 2}$ & $\mathbf{1 . 9 9 0}$ & $\mathbf{1 . 7 8 8}$ & $\mathbf{2 . 3 0 4}$ & $\mathbf{2 . 2 5 3}$ \\
\hline
\end{tabular}

Table S3. Bio-oil and water distribution in the product stream

\begin{tabular}{ccccccc}
\hline \multicolumn{7}{c}{ Bio-oil and water distribution in product stream } \\
\hline $\begin{array}{c}\text { pH in } \\
\text { feed }\end{array}$ & $\begin{array}{c}\text { pH in } \\
\text { product }\end{array}$ & $\begin{array}{c}\text { Water } \\
\text { content in } \\
\text { oil (\% wt.) }\end{array}$ & $\begin{array}{c}\text { Bio-oil } \\
\text { phase } \\
\text { (\% wt.) }\end{array}$ & $\begin{array}{c}\text { Aqueous } \\
\text { phase } \\
(\% \text { wt. })\end{array}$ & $\begin{array}{c}\text { Bio-oil } \\
\text { water free } \\
\text { phase } \\
\text { (\%) wt.) }\end{array}$ & $\begin{array}{c}\text { Light oil } \\
\text { fraction } \\
\text { (\% wt.) }\end{array}$ \\
\hline 8.9 & 7.5 & 11.4 & 4.3 & 95.8 & 3.8 & 31.0 \\
9.3 & 7.7 & 23.3 & 4.9 & 95.1 & 3.8 & 34.7 \\
9.5 & 8.1 & 51.2 & 6.8 & 93.2 & 3.3 & 30.2 \\
9.8 & 8.9 & 40.5 & 5.0 & 94.9 & 3.0 & 32.1 \\
10.1 & 9.5 & 25.2 & 5.3 & 94.7 & 3.9 & 32.0 \\
10.4 & 9.7 & 22.1 & 4.2 & 95.8 & 3.3 & 35.3 \\
\hline
\end{tabular}

${ }^{a}$ : Mass percent (wt. \%) of light oil GC-MS identified phenolic compounds in the total bio-oil.

Table S4. Total organic yields (\% wt.) in bio-oil and WSO fractions calculated on dry lignin basis (identified by GC-MS).

\begin{tabular}{lcccccc}
\hline pH in the feed & $\mathbf{8 . 9 0}$ & $\mathbf{9 . 3 0}$ & $\mathbf{9 . 5 0}$ & $\mathbf{9 . 8 0}$ & $\mathbf{1 0 . 1 0}$ & $\mathbf{1 0 . 4 0}$ \\
pH in the product & $\mathbf{7 . 5 0}$ & $\mathbf{7 . 7 0}$ & $\mathbf{8 . 1 0}$ & $\mathbf{8 . 9 0}$ & $\mathbf{9 . 5 0}$ & $\mathbf{9 . 7 0}$ \\
\hline Anisoles & 4.68 & 4.76 & 3.69 & 3.30 & 5.94 & 5.26 \\
Alkyl phenols & 7.36 & 8.14 & 6.57 & 6.90 & 12.21 & 11.63 \\
Guaiacols & 3.04 & 2.47 & 1.87 & 1.35 & 1.51 & 1.21 \\
Acetyl phenols & 0.40 & 0.35 & 0.45 & 0.34 & 0.96 & 0.88 \\
Catechols & 4.34 & 4.43 & 3.97 & 3.64 & 5.59 & 5.80 \\
Xanthenes & 0.48 & 0.58 & 0.37 & 0.36 & 0.64 & 0.54 \\
Phenolic dimers & 2.03 & 2.50 & 1.79 & 1.60 & 2.85 & 2.50 \\
Retene & 0.17 & 0.18 & 0.09 & 0.12 & 0.16 & 0.13 \\
\hline
\end{tabular}


Table S5. Elemental and ash analysis of LignoBoost Kraft lignin and bio-oil fraction (\% wt.)

\begin{tabular}{lrrrrrrr}
\hline Experiment & Lignin & \multicolumn{1}{c}{ A } & \multicolumn{1}{c}{ B } & \multicolumn{1}{c}{ C } & \multicolumn{1}{c}{ D } & \multicolumn{1}{c}{ E } & \multicolumn{1}{c}{ F } \\
\hline Ash (\%) & 0.8 & 1.4 & 1.5 & 2.3 & 3.2 & 4.0 & 4.2 \\
S & 1.85 & 0.4 & 0.4 & 0.4 & 0.4 & 0.2 & 0.2 \\
C & 65.6 & 74 & 75.3 & 75 & 74.8 & 75.7 & 75.8 \\
H & 5.7 & 6.8 & 6.4 & 5.9 & 5.7 & 6.0 & 5.9 \\
N & NA $^{*}$ & 0.1 & 0.11 & $<0.05$ & 0.1 & 0.05 & 0.1 \\
K & NA $^{*}$ & 1.3 & 1.4 & 1.9 & 2.3 & 2.9 & 3.3 \\
O** & 26 & 17.3 & 16.3 & 16.4 & 15.8 & 14.1 & 13.8 \\
Na (mg/kg) & NA $^{*}$ & 64 & 100 & 160 & 180 & 180 & 200 \\
\hline
\end{tabular}

* Not Analysed

** Calculated value.

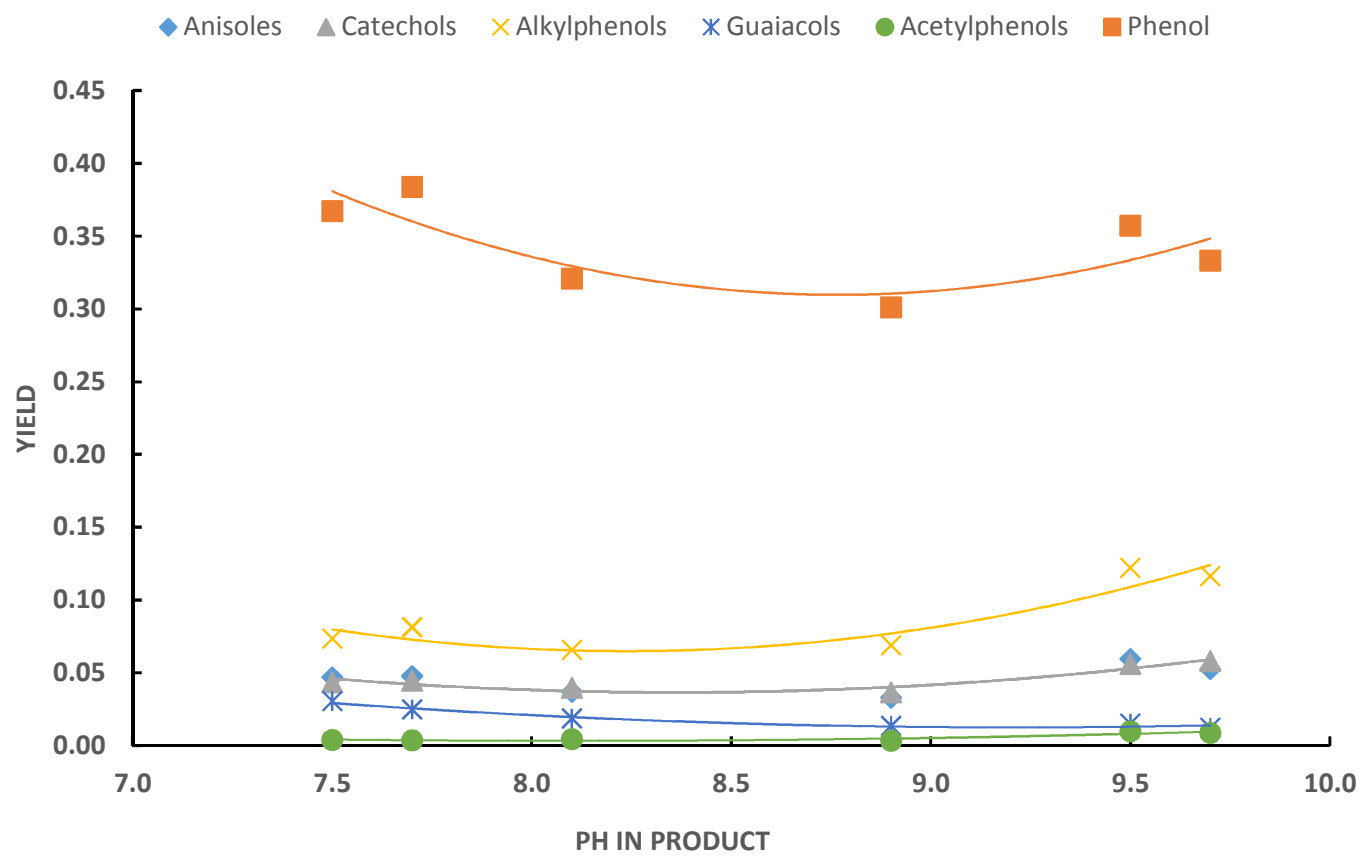

Figure S1. Total monomer yields including phenol in bio-oil and WSO fractions calculated on dry lignin basis. 